\title{
Nonlinear Response of HTSC Thin Film Microwave Resonators in an Applied DC Magnetic Field
}

\author{
Durga P. Choudhury ${ }^{a, b}$, Balam A. Willemsen ${ }^{a, b}$, John S. Derov ${ }^{b}$ and S. Sridhar ${ }^{a}$ \\ ${ }^{a}$ Physics Department, Northeastern University, Boston, MA 02115 \\ ${ }^{b}$ Rome Laboratory, Hanscom AFB., Bedford, MA 01730
}

\begin{abstract}
The non-linear microwave surface impedance, $Z_{s}=R_{s}+i X_{s}$, of patterned YBCO thin films, was measured using a suspended line resonator in the presence of a perpendicular DC magnetic field, $H_{D C}$, of magnitude comparable to that of the microwave field, $H_{r f}$. Signature of the virgin state was found to be absent even for relatively low microwave power levels. The microwave loss was initially found to decrease for small applied $H_{D C}$ before increasing again. Also, non-linearities inherent in the sample were found to be substantially suppressed at low powers at these applied fields. These two features together can lead to significant improvement in device performance.
\end{abstract}

\section{INTRODUCTION}

The microwave response of high- $\mathrm{T}_{c}$ superconductors (HTSC) is important both from the point of view of microwave applications of HTSC[1] and fundamental physics[2]. An understanding of the loss mechanisms, field and current profiles and nature of non-linearities can lead to improvement in fabricated devices that use them. While numerous experimental studies of non-linear microwave response of HTSC have been done [3], none of them to our knowledge have probed the non-linear response in the presence of DC magnetic fields, where the DC and microwave fields are of comparable magnitude. In this situation the effect of the microwave field cannot be considered as a small perturbative Lorentz force on the vortex lattice generated by the DC field, as is often done at high fields[4]. Such a situation can also act to test various models that have been proposed for the non-linear response.

\section{EXPERIMENTAL TECHNiques}

We used a patterned suspended resonant thin film of $\mathrm{YBa}_{2} \mathrm{Cu}_{3} \mathrm{O}_{7-\delta}$ housed in a rectangular copper package to carry out this series of experiments. Similar methods have been used before[5], [6] with great success to investigate vortex dynamics and non-linearities in HTSC. The film, procured from Neocera Inc, was deposited on a 0.6 in $\times$

Manuscript received August 27, 1996.

D. P. Choudhury, 617-373-2948, fax 617-373-2943, dpc@neu.edu, http://sagar.physics.neu.edu/

Work at Northeastern University was supported by the AFOSR through Rome Labs, Hanscom AFB.
0.22 in $\times 0.010$ in $\mathrm{LaAlO}_{3}$ substrate by laser ablation, and was subsequently patterned in-house to a straight line of dimension 0.56 in $\times 0.004$ in using methods described elsewhere [7]. In order to obtain the highest possible $Q^{\prime} \mathrm{s}$, the package was mechanically polished and thoroughly cleaned before each set of experiments. The resonator was made symmetric by placing a blank substrate of the same material and dimensions on top of the film before it was loaded into the package.

The assembly, complete with a controlling heater and temperature sensor, was inserted into the sample chamber of a Cryo Industries Variable Temperature cryostat. Two independent carbon glass sensors and temperature controllers were used to stabilize the temperature of the cavity to the degree required for these experiments. A LakeShore DR91C was used for gross control of the vaporizer temperature, which was set slightly below the desired sample temperature. The desired sample temperature was obtained and finely controlled with a LakeShore DR93CA. Temperature stability of the order of $1 \mathrm{mK}$ were typical for the experiments presented here, where the data took up to two hours to obtain for each run.

DC magnetic fields up to 1000 Oe was applied parallel to the $c$-axis of the sample using a custom-built Walker Scientific copper solenoid and a LakeShore 622 superconducting power supply. Unlike typical Helmholtz coil configurations, the solenoid has no polecaps, thus ensuring that there is no remanent field, save for possibly the geomagnetic fields. It is worth pointing out that our experiment does not use any superconducting ground planes unlike parallel plate or microstrip resonators, thus avoiding complications due to demagnetization effects from such plates.

Microwaves were inductively coupled to and from the resonator by means of loops at the ends of stainless steel coaxial lines. The microwave transmission amplitude $S_{21}$ was then measured using an HP 8510C Automatic Network Analyzer. The coupling strength was adjusted by varying the distance between the loops and the resonator as well as their relative orientation. Coupling could thus be reduced to the point that the loaded and unloaded $Q^{\prime}$ s are indistinguishable, simplifying the data extraction process. At the low input power levels that were used to carry out these experiments, the trace was very noisy. This coupled with the fact that we require extreme sensitivity to very small changes mean that we could not simply determine the $Q$ from the maximum frequency and $-3 \mathrm{~dB}$ bandwidth as is often done. In order to reduce the noise 


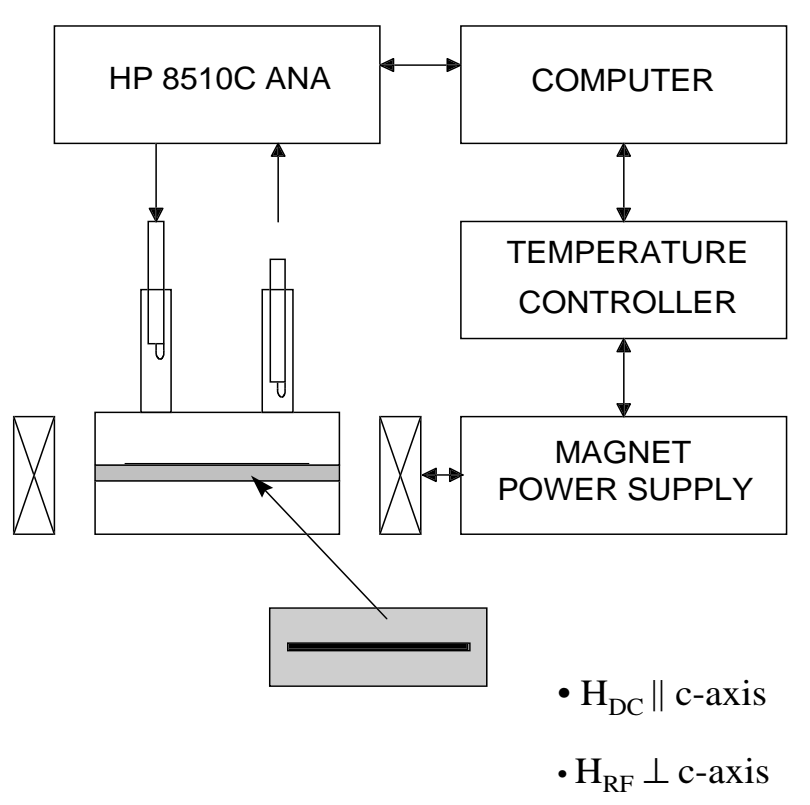

Fig. 1. Block diagram of Experimental Setup

and obtain the required sensitivity:

- The network analyzer was used in the "Step" mode, in which every frequency point is individually synthesized

- The signal was heavily averaged to get rid of the random noise,

- The trace was fitted using the method of least squares to Lorentzian shape, and

- The frequency span was kept as narrow as possible, usually only about $20 \%$ larger than the $-3 \mathrm{~dB}$ bandwidth.

The center frequency and the $-3 \mathrm{~dB}$ bandwidth obtained from the fit agreed very well with those directly read off the trace, especially at low power levels where the trace is closest to Lorentzian shape, but provided significantly enhanced sensitivity to small changes.

\section{Results And Discussion}

Ubiquitous intrinsic non-linearities have been observed in thin film specimens of High- $\mathrm{T}_{c}$ materials[8], [6], and some aspects of these non-linearities appear to be explained by a current-induced critical state model[9]. The present experiments, which involve both microwave and DC fields of comparable magnitude so as to study the interplay of these two effects, were designed to further test these critical state and other ideas. Our experiments show that the presence of even relatively low microwave powers can induce vortices in the film, emulating the response of a DC field. The signature of this fact come from the observation that low DC field hysteresis does not show the virgin state response.

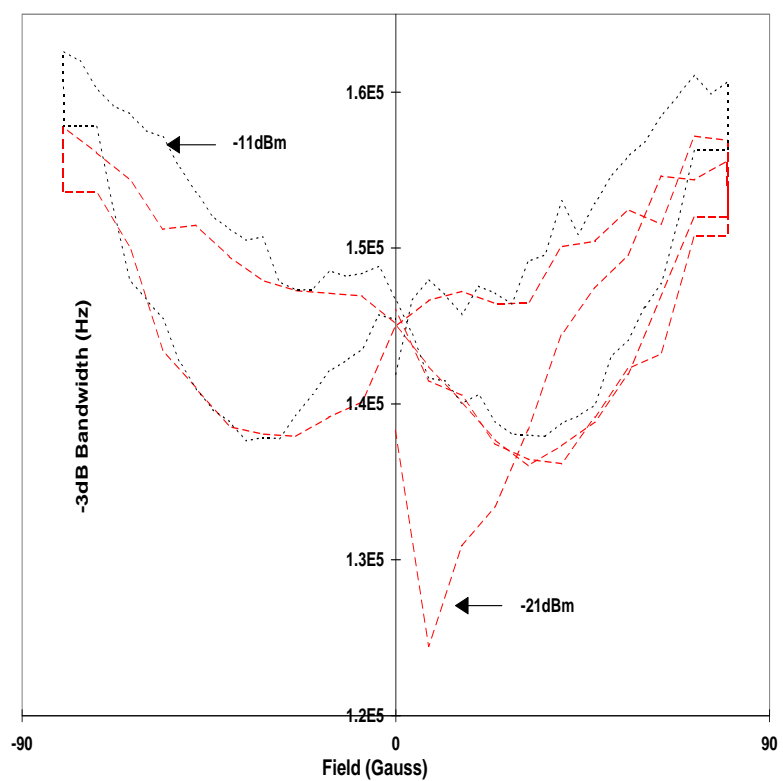

Fig. 2. Low field hysteresis at $-21 \mathrm{dBm}$ and $-11 \mathrm{dBm}$ of input power, at $10 \mathrm{~K}$. Notice the absence of the "tail" corresponding to virgin response at higher powers. In order to highlight the similarities between the two plots, they have been superposed on each other by adding a constant of $10 \mathrm{kHz}$ off the $-21 \mathrm{dBm}$ power plot.

In a typical sample, the signature of the virgin state (i.e. absence of trapped flux tubes) in the low DC field hysteresis experiments manifests itself as a sharp rise in the $-3 \mathrm{~dB}$ bandwidth as field is slowly increased from zero corresponding to initial penetration of flux. As the field is further increased to a value $H_{\max }$ and then cycled between $H_{\max }$ and $-H_{\max }$, where $H_{\max }$ is a field of the order of a few hundred Gauss, this initial behavior is never reproduced; instead, it goes through a butterfly-shaped hysteresis loop[10].

The same experiment, performed on the films under discussion, yields two new observations :

- The initial "virgin" response vanishes at higher microwave powers. This seems to indicate that microwave fields can create enough vortices in in the sample to wash away the virgin state response, mimicking the effect of an applied DC field.

- A sharp dip in $R_{s}$ is observed at a field scale $H_{D C} \sim$ $5 \mathrm{G}$ in the virgin response, indicating that a small applied DC field serves to lower $R_{s}$.

The second result was verified when we did a measurement of $R_{s}$ against applied microwave power in a fixed $H_{D C}$. The decrease in $R_{s}$ reproduces itself, as is evident from fig.3. Another observation from the microwave power ramp experiments is that the non-linearities in the sample also get substantially suppressed at these low field for low microwave powers. As $H_{D C}$ is increased, $R_{s}$ gradually rises and finally goes above it's zero field value. To further ensure that this observation is genuine and 


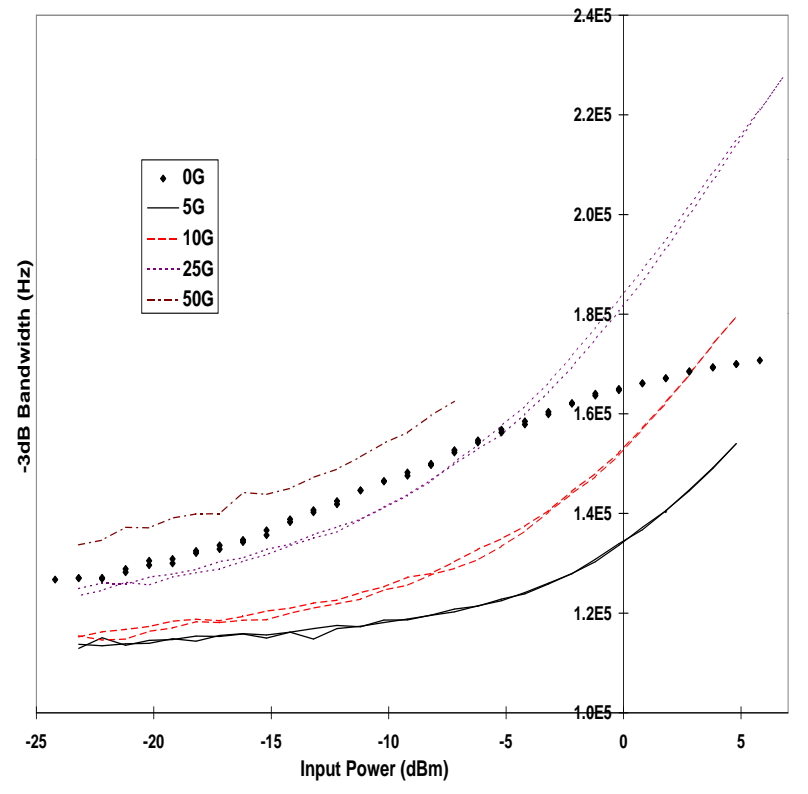

Fig. 3. Typical power dependence of resonance widths, taken at $10 \mathrm{~K}$

is not an artifact of some experimental inconsistency, we repeated the measurements on a film patterned out of a different albeit similar wafer. Although results obtained from this film were not quantitatively identical with those of the other, which would be expected because of the differences on growth, deposition and patterning of the two films, the two characteristic features described above was observed to a comparable extent in the second film. Also to rule out the effect of any stray remanent DC field, we carried out the microwave power ramp measurements with the DC field reversed and no such effects were found.

We have examined the present data in the framework of two proposed explanations for non-linear response in HTSC, viz. weak links and dynamics of a current-driven critical state. The weak link picture can be viewed in terms of a resistively shunted junction (RSJ) model, taking the effect of the DC field to be a DC current flowing on the surface in addition to the rf current. The equation of motion for the relative phase between the coupled grain then become $\dot{\phi}+\sin \phi=i_{d c}+i_{\omega} \cos \omega t$. The dynamic impedance can be calculated from $Z_{\omega}=\dot{\phi}_{\omega} / i_{\omega}$. While numerical calculations of this response yield very interesting effects as $i_{d c}$ is varied, this approach does not seem to yield the results that are observed in this experiment.

The critical state model should also lead to ac $+\mathrm{dc}$ effects, so that the non-linearity should be modified by the DC magnetic field. However a calculation of this effect is not straight-forward, since it requires a prescription for the present case where the loss needs to be calculated when $i_{r f}$ is varied over on rf cycle for finite $H_{D C}$. The available prescription in the literature [11] does not consider this case, but instead considers a different method of varying $i_{r f}$ and $H_{D C}$. Hence, at the present, it is not pos- sible to determine if the critical state model can explain these unusual results.

It is worth noting that the unusual decrease in $R_{s}$ observed here can occur due to non-equilibrium effects and in fact have been seen in low $T_{c}$ superconductors [12]. There it was shown that when the microwave frequency $\omega>\tau^{-1}$, where $\tau$ is the quasiparticle relaxation time, a non-equilibrium quasiparticle distribution can occur which leads to a decrease of $R_{s}$ in the presence of an $i_{d c}$. Another related phenomenon which occurs is an enhancement of the superconducting gap. While this condition is met in pure metals at low temperatures, it is not clear if this happens in the high $T_{c}$ materials.

However it is interesting to note that in $\mathrm{YBa}_{2} \mathrm{Cu}_{3} \mathrm{O}_{7-\delta}$ crystals, $R_{s}(T)$ is non-monotonic and there are regions of temperature where $\left(\partial R_{s} / \partial T\right)<0$. This unusual, apparently non-thermodynamic result, may imply that $\left(\partial R_{s} / \partial i_{d c}\right)<0$ need not be surprising.

\section{Conclusion}

We have described a novel effect in which both the microwave losses and non-linear response decrease in the presence of small magnetic fields. Although a clear explanation of this effect is lacking, and it could arise from non-equilibrium quasiparticle effects, the present observation implies that losses can be reduced by as much as $30 \%$ and could have interesting implications for device performance.

\section{REFERENCES}

[1] Zhi-Yuan Shen and Charles Wilker, "Raising the powerhandling capacity of hts circuits", Microwaves $\& R F$, pp. 129-138, April 1994.

[2] T. C. L. Gerhard Sollner, Jay P. Sage, and Daniel E. Oates, "Microwave intermodulation products and excess critical current in $\mathrm{YBa}_{2} \mathrm{Cu}_{3} \mathrm{O}_{7-\delta}$ josephson junctions", Appl. Phys. Lett., vol. 68, no. 7, pp. 1003-1005, February 1996.

[3] Charles Wilker, Zhi-Yuan Shen, Philip Pang, Willam L. Holstein, and Dean W. Face, "Nonlinear effects in high temperature superconductors: 3rd order intercetpt from harmonic generation", IEEE Trans. Appl. Supercond., vol. 5, no. 2, pp. 1665, June 1995.

[4] Mark W. Coffey and John R. Clem, "Theory of rf magnetic permeability of isotropic type-II superconductors in a parallel field", Phys. Rev. B, vol. 45, no. 17, pp. 9872, May 1992.

[5] Balam A. Willemsen, John S. Derov, José H. Silva, and S. Sridhar, "Vortex dynamics at microwave frequencies in patterned $\mathrm{YBa}_{2} \mathrm{Cu}_{3} \mathrm{O}_{7-\delta}$ thin films", Appl. Phys. Lett., vol. 67, no. 4, pp. 551-553, July 1995.

[6] Balam A. Willemsen, John S. Derov, José H. Silva, and S. Sridhar, "Nonlinear response of suspended high temperature superconducting thin film microwave resonators", IEEE Trans. Appl. Supercond., vol. 5, no. 2, pp. 1753-1755, June 1995.

[7] Balam A. Willemsen, Vortex Dynamics at high Frequencies in Layered Superconductors, PhD thesis, Northeastern University, Boston, MA 02115, October 1995.

[8] P. P. Nguyen, D. E. Oates, G. Dresselhaus, and M. S. Dresselhaus, "Nonlinear surface impedance for $\mathrm{YBa}_{2} \mathrm{Cu}_{3} \mathrm{O}_{7-\mathrm{x}}$ thin films: Measurements and a coupled-grain model", Phys. Rev. $B$, vol. 48, no. 9, pp. 6400-6412, September 1993.

[9] S. Sridhar, "Non-linear microwave impedance of superconductors and ac response of the critical state", Appl. Phys. Lett., vol. 65, no. 8, pp. 1054-1056, August 1994. 
[10] J. S. Derov Balam A. Willemsen and S.Sridhar, "Critical state flux penetration and linear microwave vortex response in $\mathrm{Y}_{1} \mathrm{Ba}_{2} \mathrm{Cu}_{3} \mathrm{O}_{7-\delta}$ films", Unpublished.

[11] Ernst Helmut Brandt and Mikhail Indenbom, "Type-IIsuperconductor strip with current in a perpendicular magnetic field", Phys. Rev. B, vol. 48, pp. 12893-12906, 1993.

[12] Srinivas Sridhar, Microwave Dynamics of Quasiparticles and Critical Fields in Superconducting Films, PhD thesis, California Institute of Technology, 1983. 